\title{
Genetic investigation of 211 Chinese families expands the mutational and phenotypical spectrum in hereditary retinopathy genes through targeted sequencing technology
}

\section{Zhouxian Bai}

The First Affiliated Hospital of Zhengzhou University https://orcid.org/0000-0001-7071-666X

\section{Zhouxian Bai}

The first affiliated Hospital of Zhengzhou University

Yanchuan Xie

First Affiliated Hospital of Henan University of Science and technology

\section{Lina Liu}

First Affiliated Hospital of Zhengzhou University

\section{Jingzhi Shao}

Southern Medical University Nanfang Hospital

Yuying Liu

First Affiliated Hospital of Zhengzhou University

\section{Xiangdong Kong ( $\square$ kongxd@263.net)}

Genetics and Prenatal Diagnosis Center, The First Affiliated Hospital of Zhengzhou University, Zhengzhou, Henan 450052, China

https://orcid.org/0000-0003-0030-7638

\section{Research article}

Keywords: hereditary retinopathy, novel mutations, targeted sequencing, genetic testing

Posted Date: April 8th, 2020

DOl: https://doi.org/10.21203/rs.3.rs-20958/v1

License: (c) (i) This work is licensed under a Creative Commons Attribution 4.0 International License. Read Full License

Version of Record: A version of this preprint was published at BMC Medical Genomics on March 29th, 2021. See the published version at https://doi.org/10.1186/s12920-021-00935-w. 


\section{Abstract}

Background: Hereditary retinopathy is a significant cause of blindness worldwide. Despite the discovery of many mutations in various retinopathies, a large part of patients remain undiagnosed genetically. Targeted next generation sequencing of the human genome is a suitable approach for retinopathy molecular diagnosis.

Methods: We describe a cohort of 211 families from central China with various forms of retinopathy investigated using a NGS multi-gene panel as well as Sanger sequencing. We validated the candidate variants by PCR-based Sanger sequencing. We have made comprehensive analysis of the cases through sequencing data and ophthalmologic examination information.

Results: The potentially causal mutation was identified in majority of the families with retinopathy ( $57.9 \%$ of 95 families) and Leber hereditary optic neuropathy (LHON) (21.6\% of 116 families). The identified mutations (68mutations, 37 of which are novel) of the 95 families spanned 31 known disease genes, about half have not been reported relation to hereditary retinopathy. This study, the NGS panel solution provides $45.3 \%$ potential diagnostic rate of the retinopathy families and another $12.6 \%$ families receive candidate gene mutations with undefined pathogenicity.

Conclusion: Our study showed novel mutations and phenotypic aspects of retinopathy, and demonstrated genetic and clinical heterogeneity of the conditions. Our results illustrated the significance of molecular genetic testing for patients with hereditary retinopathy.

\section{Background}

Hereditary retinopathy is one of the most common genetic blinding retinal diseases. Retinopathy is highly heterogeneous in hereditary and clinical phenotypes. The main inheritance patterns include autosomal dominant, autosomal recessive inheritance and X-linked inheritance. The hereditary retinopathy mainly includes retinitis pigmentosa, macular degeneration, Leber's hereditary optic neuropathy (retina inconvenienced early stage) and retinal dysplasia involved in this study. Retinitis pigmentosa (RP) is a group of hereditary blinding fundus diseases caused by abnormalities in photoreceptors of the retina[1]. Its main clinical features are progressive visual field defect, night blindness, bone spicule like pigmentation and abnormal electroretinogram. Leber hereditary optic neuropathy (LHON) is a mitochondria hereditary eye disease that involves with retinal ganglion cells resulting eventually in degeneration and atrophy of optic nerve[2]. With the popularization and clinical application of gene sequencing technology, more and more disease-causing genes and its mutations have been discovered, and these genes are mainly expressed in photoreceptor cells and retinal pigment epithelial cells[3]. A good understanding of retinopathy genes not only provides theoretical basis for diagnosis and genetic counseling, but also supports guidance for gene therapy of the diseases[4].

The study of retinopathy genetics is important because that it improves our understanding of the molecular aspects of eye development, disease and treatment. Despite the discovery of pathogenic mutations and genes of various types of retinopathy (such as RP), there are many unknowns waiting to be found. Hence, targeted next generation sequencing (NGS) of the human genome in related Chinese families and patients expanded the mutational spectrum and deepened our understanding of the mechanism of disease. This investigation also increased the understanding of heterogeneity of clinical manifestations of diseases and enriched the phenotypic spectrum of diseases.

\section{Methods}

\section{Subject collection}

There were 211 Chinese families with retinopathy from central China recruited for this study, included 116 patients from different families with chief complaints of monocular or binocular fundus optic atrophy (LHON suspected) and 95 families with chief complaints of other retinopathy. The 116 patients receiving LHON Sanger sequencing with fundus optic atrophy, and the 95 patients receiving targeted NGS with other retinopathy have been examined and diagnosed by ophthalmology. Specific clinical manifestations of patients are shown in the results part of the paper. Sample was obtained with written informed consent. The retinopathy patients came for medical and genetic consultations in our hospital during 2017 and 2019, $4 \mathrm{~mL}$ peripheral blood were drawn from the persons in the 211 retinopathy families respectively. Amniotic fluid of the mothers in corresponding families was extracted for prenatal diagnosis (see in Figure S25). The clinical materials of the patients included this study were collected when they got medical service at clinic.

\section{Targeted Next Generation Sequencing and Sanger Sequencing}

Genomic DNA was extracted from EDTA-treated blood samples using Blood DNA Midi Kit D3494 (Omega Bio-tek, USA) through nucleic acid automatic extraction equipment (Eppendorf epMotion 5075m, Germany). Amniotic fluid cell DNA was extracted and cleaned using QIAamp Blood DNA Midi Kit (250, Germany) and Genomic DNA Clean \& Concentrator (Zymo Research, USA).

A customized panel (MyGenostics, Beijing China) captured 463 known genes (Table S1) related with retinal disease was designed to detect the genetic cause of the congenital retinopathy families. The panel sequencing was conducted on the Illumina NextSeq500 system in our clinical lab. Version GRCh37 is the human reference genome used for short reads mapping. PCR-based Sanger sequencing was used to validate the variants 
which chosen as disease causing mutations through NGS. The carrying situation of the novel mutations of their family members was also tested by Sanger sequencing. The PCR primers were designed by GeneTool software. Capillary electrophoresis apparatus (ABI 3130xI, USA) and dGTP BigDye ${ }^{\circledR}$ Terminator sequencing kit (ABI, USA) were used for Sanger sequencing.

The patients with chief complaints of LHON were tested thorough PCR-based Sanger sequencing, which included 3 common mutant sites such as $\mathrm{mt} .3460$, mt.11778 and mt.14484, and 10 rare mutant sites such as mt.3376, mt.3635, mt.3700, mt.3733, mt.14482, mt.14495, mt.14502, mt.14568, mt.14498, mt.14325. The PCR primers were designed by GeneTool software (Table S2).

\section{Population control}

Frequency of the detected mutations in the population was investigated by querying the Genome Aggregation Database (gnomAD, http://gnomad-old.broadinstitute.org/) because of its wider large-scale sequencing data resource. We chose the frequencies of mutation sites in all populations and in East Asian populations as controls.

\section{Functional prediction analysis}

Retrieve the candidate pathogenic mutation sites in the public databases which included dbSNP, 1000G and ExAC, and check them whether been recorded. We also plan to retrieve the candidate sites in HGMD (The Human Gene Mutation Database at the Institute of Medical Genetics in Cardiff) professional to ensure their pathogenicity. PhyloP and PhastCons software were used to analyse the conservation of corresponding amino acid sequence of the missense mutated locus. Pathogenic analysis was conducted by SIFT, PolyPhen_2 and Mutation t@sting online tools. We also analyzed the second structure, disorder region and mutation effect of the missense mutated site by Predictprotein online tool. Three dimensional structure construction of target protein sequence was using Swiss-Model protein model structure simulation software.

\section{Results}

The genome variation results of different patients and their families were classified and summarized by pathogenic genes.

\section{Mutation distribution in suspected LHON patients}

In two and one half years, 116 suspected patients with optic neuropathy or LHON were examined to help diagnose 25 cases of LHON (seen in Figure 1) among them. The ratio of male to female in patients with LHON is 4/1 in our investigation. The average age of the patients diagnosed as LHON was 19 years old, their age ranged from 6 to 36 years old. The three common mutant sites of mt.3460, mt.11778 and mt.14484 are main (96\%) causes of LHON, and MTND4 m.11778G >A is the most common pathogenic mutation among them, then MTND6 m.14484T>C and MTND1 m.3460G>A. Just one rare mutant that MTND6 m.14502T>C was found in these Chinese patients from central China. Several LHON patients are with incomplete mitochondrial mutation or two mutations.

\section{Pathogenic mutations of the hereditary retinopathy}

In the same period, 95 families were examined by using the targeted sequencing technology, which are suspected with retinitis pigmentosa or congenital retinopathy. Partial genealogical trees are shown in Figure S25. We totally identified 68 distinct mutations from 31 different known disease genes in the patients of these families, 37 mutations are novel among them. The results are grouped by related genes found in the retinopathy patients. In this investigation, 57.9\% of the families under test have been detected significant mutants (Table 1 and Table 2 ). The mutations listed in Table 1 are predicted as damaging or disease causing by function analysis software, and a part of the mutations have been studied and reported. The phenotype and mutation of each of these families are co-segregation, respectively. Targeted sequencing of retinopathy related genes provides $45.3 \%$ diagnostic rate, and another $12.6 \%$ families receive candidate gene mutations with undefined pathogenicity in this study.

Four families (Family 14, 15, 48 and 54) suffering from retinitis pigmentosa caused by $R H O$ mutations, the patients of these families presented with night blindness from childhood, visual field defect or tubular visual field and retinitis pigmentosa. The Sanger sequencing results of mutant sites of Family 14 and Family 15 are presented in Figure $\mathbf{S 1 3}$ and $\mathbf{S 1 4}$ respectively. NDP mutations can lead to familial exudative vitreoretinopathy or Norrie disease. Two families (18 and 46) with FEVR2 have been detected two novel NDP mutations, c.124C>A (p.H42N) (Sanger result in Figure 3) and c.401_402delGA (p.*134Wfs*13). The probands were found that their eyes do not chase things when they a few months old, and no blood vessel area of binocular fundus detected by ophthalmoscopic examination. Male patients of the two families have no other serious visual problems. Two families (32 and 55) diagnosed as Norrie disease have two known NDP mutations, c.343C>T (p.R115X) and c.268C>T (p.R90C). The two months old male patient in Family 32 has vitreous hyperplasia, right microphthalmos and microcorneas, while the male patient in Family 55 is total blindness and atrophy of eyeballs. Mutation of USH2A can cause retinitis pigmentosa with or without syndromic hearing loss. The patients from Family 7 and Family 47 with Usher syndrome, type 2A, they presented with retinitis pigmentosa and hearing impairment. The two patients have different mutations in USH2A gene (Figure S11, S17 and S18). The patient of Family 9 with USH2A mutation just has nonsyndromic retinal diseases. Three families (Family 27, 38 and 51) were detected different RS1 hemizygous mutations in the 
retinoschisis patients. The results of fundus examination and optical coherence tomography of the congenital retinoschisis patient in Family 38 are shown in Figure S5 and S6. Patients from the two families (Family 1 and 2) can be diagnosed as RP, 38 caused by MERTK gene mutations. These patients are characterized by retinitis pigmentosa, night blindness and visual field loss.

Small deletion and nonsense mutation of CYP4V2 resulted in Bietti crystalline corneoretinal dystrophy of the patients from Family 3 and 4 . The Patient's result of visual electrophysiology in Family 3 is shown in Figure S1. The CYP4V2 c.(802-8)_810delTCATACAGGTCATCGCTinsGC and c.958C > T mutations in Family 4 are shown in Figure S8 and S9. FSCN2 c.72delG causes retinitis pigmentosa, type 30 in two unrelated families (Family 5 and 6). FSCN2 c.72delG Sanger sequencing result is shown in Figure S10. Small deletion and frameshift mutation of PRPF31 resulted in retinitis pigmentosa, type 11 of the patients from Family 12 (PRPF31 c.1074-8_1079delGTACCGGTCCCCAG novel mutation seen in Figure S12) and Family 50. There are four RP patients from three generations of the Family 12. In addition to the symptoms of retinitis pigmentosa, night blindness and tubular visual field, the proband and his father (Figure S25) were also treated with bilateral cataract surgery. There are two families (Family 33 and 52) with family history of RP and night blindness caused by the same mutation, RPGR c.2236_2237delGA. There are two families (Family 43 and 44) with family history of RP and night blindness caused by the different mutations of $R P 2$, and the splicing mutation c.769-2A>G has been reported to pathogenic for RP, the frameshift mutation c.572_582dup11 is novel.

There seventeen families affected by different retinal diseases have been detected pathogenic or likely pathogenic mutations of 17 different related genes. The patient of Family 36 with macular degeneration has poor eyesight. The patient of Family 16 has retinochoroidal coloboma, his results of visual field examination and mutation sequencing is shown in Figure S4. Sanger sequencing result of the mutant site in Family 17 is shown in Figure S15, and the RP proband also combined with cataract when he twenty-six years old. The patient of Family 20 was two years old (Sanger result seen in Figure S20), her full field ERG showed that rod cells had no waves, while scotopic ERG showed that the amplitudes ofaand $\beta$ waves decreased. The ophthalmoscopic image and sequencing result of RCS patient from Family 22 are presented in Figure S19. The CNGA1 mutations in Family 45 were validated by Sanger sequencing (Figure S16). The thirty-four years old mother and her daughter have macular degeneration of fundus in Family 34. The forty-one years old patient of Family 35 suffered from retinal detachment, primary vitreous hyperplasia and familial exudative vitreoretinopathy, and his mother with the same $F Z D 4 \mathrm{c} .612 \mathrm{~T}>\mathrm{A}$ heterozygous mutation also had the same eye symptoms. Both of the thirty-three years old man and his mother with neurodeatrophia and familial exudative vitreoretinopathy carry the $\angle R P 5$ c.485_488delACGG heterozygous mutation in Family 37. The three years old girl with congenital horizontal nystagmus has compound heterozygous variation of SLC38A8 in Family 39, and her parents are carriers of the heterozygous variation. The two years old boy is a Leber congenital amaurosis patient, and his parents are the carriers of heterozygous variation of AIPL 1 in Family 40. The hemizygous FRMD7 c.910C>T (p.R304X) mutation leaded to Nystagmus of the boy in Family 41, and his mother carries the heterozygous mutation. The five years old boy diagnosed as Leber congenital amaurosis caused by homozygous mutation of GUCY2D c.3177_3178delAC inherited from parents.

\section{Variants of undetermined significance in retinopathy families}

The mutations listed in Table 2 are predicted as damaging or associated with the clinical phenotypes of the families, which can be considered as candidate mutants but not fully determined. The families included in Table 2 usually have no family history of hereditary diseases. There are four families $(8,10,23$ and 53) been detected different compound heterozygous mutations of USH2A, the mutations associate with the nonsyndromic phenotype of retinitis pigmentosa of these patients without obvious hearing impairment. The mutations found in the four families are likely pathogenic. The four years old boy in Family 19 was detected compound heterozygous mutation of USH1C, which gene variation can cause Usher syndrome -type 1C characterized as severe hearing impairment and retinitis pigmentosa. The patient with RP and night blindness has suffered from bilateral secretory otitis media, but his bilateral hearing is basically normal. His both ears passed TEOAE (transient evoked otoacoustic Emissions) examination and DPOAE (distortion product otoacoustic emissions) test at the acoustic frequency (1k, 2k, 4k and 8k $\mathrm{Hz}$ ), but left ear did not pass DPOAE at $0.5 \mathrm{k} \mathrm{Hz}$ acoustic frequency. I wave latency was slightly longer after $80 \mathrm{dBnHL}$ short-tone stimulation in ABR (auditory brainstem response) test of the boy, and other waves were normal. So the USH1C mutation is associated with the phenotypes, but undetermined significance. The other patients from different families (13, 25, 56, 24, 29 and 31) (Table 2) have candidate gene mutations and corresponding phenotypes, respectively. The Sanger sequencing results of Family 56 is shown in Figure S21 and S22. It should be noted that the RS1 c.240G>C (p.Q80H) mutation not co-separated from the phenotype and genotype in Family 26.

\section{Prenatal diagnosis of families with confirmed patients}

There are 11 families with patients confirmed on clinical and genetic level subjected to prenatal molecular diagnosis. The fetuses from Family 15 and 43 were diagnosed as RP on gene level, they then terminated the pregnancy. The fetus from Family 18 was diagnosed as FEVR2 on gene level, but the mother decided to continue pregnancy due to the clinical heterogeneity of the disease. The baby of Family 18 was born as FEVR2 with mild phenotype. The Family 2 obtains healthy offspring through prenatal diagnosis and the third generation of IVF technology. There five families (Famliy 17, 33, 44, 49 and 55) have healthy offsprings through natural pregnancy and prenatal diagnosis. The heterozygous carriers born from two families (Family 42 and 46) are not presented clinical phenotypes of the diseases, respectively.

\section{Specific cases}


Family 2: There are two RP patients in Family 2. The thirteen years old sister with patchy defects of visual field and abnormal ERG, she has homozygous mutation of MERTK c.754delC (Figure 4). The ten years old brother's symptoms was milder than his sister, he also had defects of visual field (Figure S7) and carried the same homozygous mutation. They all suffered from night blindness and visual impairment. Their parents are the carriers of the heterozygous variation of MERTK c.754delC. According to ACMG guidelines, the novel frameshift mutation of MERTK c.754delC should be considered as pathogenic, and its grade (PVS1) is high. This family acquired a healthy boy through three generations of IVF technology (pre-implantation genetic diagnosis).

Family 3: The patient with homogeneous mutation of CYP4V2 c.(802-8)_810delTCATACAGGTCATCGCTinsGC has retinitis pigmentosa and visually impaired. This mutation is known pathogenic for Bietti crystalline corneoretinal dystrophy (Bietti CCD), it involves small deletion and insertion in splicing regions. She has the typical fundus and visual electrophysiological symptoms (Figure S24 and Figure S1). So the patient can be diagnosed as Bietti CCD by combining ocular manifestations and gene mutation.

Family 5: The RP patient in Family 5 all carried known pathogenic mutation, FSCN2 c.72delG. The proband has the typical fundus and visual electrophysiological symptoms (Figure $\mathbf{S 2 3}$ and Figure S2). This mutation is the same genetic cause for Family 6 , and it is a common pathogenic mutation for RP, 30 .

Family 11: The three years old boy, one of fraternal twins, sought medical advice for night blindness. The patient's clinical manifestations also included retinal abnormalities, lateral nystagmus and finger stimulating eyeball phenomenon. He has the TULP1 compound heterozygous mutation of c.1318C>T (p.R440X) and c.1142T>G (p.V381G), his parents are heterozygous variants carrier of each of the two mutations. The nonsense mutation c. $1318 \mathrm{C}>\mathrm{T}$ ( $\mathrm{p} . \mathrm{R} 440 \mathrm{X}$ ) is known pathogenic for Leber congenital amaurosis, type 15, and the missense mutation c.1142T>G (p.V381G) is novel. c.1142T>G can lead to amino acid substitution of the protein product, it affects the function. The eye examination and mutations are presented in Figure 2. So the boy was diagnosed as LCA 15 by combining clinical manifestations and gene mutation.

Family 18: The one years old boy's fundus photographs and mutation sequencing results are shown in Figure 3. The cornea was clear in both eyes, the anterior chamber was preserved and the lens was transparent. The fundus examination showed that there was no blood vessel area in both eyes. The temporal epiretinal membrane of the right fundus vascular arch pulled the macula. The mutation of NDP can lead to familial exudative vitreoretinopathy, type 2 (FEVR2), and c. $124 \mathrm{C}>\mathrm{A}(\mathrm{p} . \mathrm{H} 42 \mathrm{~N})$ is novel for FEVR2. There is one known pathogenic mutation of c.125A>G (p.H42R) at the same location of polypeptide chain of this novel variant. According to ACMG guidelines and related prediction software, the c. $124 \mathrm{C}>\mathrm{A}(\mathrm{p} . \mathrm{H} 42 \mathrm{~N})$ should be pathogenic. FEVR2 is characterized by no blood vessel area of fundus, but severity of the disease varies. There three persons with c.124C>A (p.H42N) mutation have no blood vessel area in both fundus from this family.

Family 21: This is a consanguinuous marriage family of Chinese Hui (A Chinese minority). The patient presented with retinoschisis, macular edema and night blindness, and he carried the homozygous mutation of NR2E3 c.925C>T (p.R309W). The ophthalmological examination and mutation sequencing results of the patient are shown in Figure 5 and Figure S3. The missense mutation c.925C>T of NR2E3 is novel for Goldmann-Favre syndrome, but the c.925C>G (p.R309G) at same location of mRNA and polypeptide chain is known pathogenic for GoldmannFavre syndrome and Enhanced S-cone syndrome[5]. Some scholars believe that the severe type of Enhanced S-cone syndrome is Goldmann-Favre syndrome[6]. The patient's condition has worsened over the past 10 years, and he can be diagnosed as Goldmann-Favre syndrome by combining phenotype and genotype.

\section{Discussion}

Using targeted NGS technology and Sanger sequencing, we investigated the mutation profile and clinical features in 211 Chinese families with hereditary retinopathy during the three years. Ninety-five families were studied by targeted next-generation sequencing, and fifty-five of them had meaningful positive findings. One hundred and sixteen patients from different families were tested through Sanger sequencing, and twenty-five persons of them detected related mitochondrial mutations. Hereditary retinopathy covers a group of genetically and clinical highly heterogeneous disorders. Targeted NGS analysis is a valuable method for molecular genetics diagnostics of these diseases supported by previous studies[7-9]. These studies show that the potential molecular genetics diagnostic rate of targeted sequencing is between 38\%[8] and 76\%[9], Jespersgaard's report indicats that the detection rate of related genotypes was $72 \%$, and the detection rate of causative variants was $48 \%$ [7]. Our study provides $45.3 \%$ potential diagnostic rate of the hereditary retinopathy families and $58 \%$ meaningful detection rate of the subject families in totally. The rate of mutation found is probably comparable with the previous literatures. DNA sequencing has become a powerful diagnostic tool for hereditary retinal disease.

Twenty-five patients with positive results of mitochondrial gene testing in our study were 19 years old on average, with a male-to-female ratio of 4/1. It indicates that the patients from Chinese population have younger age and higher sex ratio than Europe and America, by comparisons with previous studies $[10,11]$. Our study did not found new mitochondrial variant, it showed that the mt.11778 and mt.14484 are most common pathogenic mutations for LHON. The possibility of over represented in LHON diagnosis is due to the smaller scale and single center collection. 
Retinitis pigmentosa is a hereditary progressive retinopathy. It is the most common group of blindness fundus diseases characterized as nocturnal blindness and progressive visual field defect caused by degeneration of retinal photoreceptor cells and pigment epithelial cells[12]. Its inheritance modes include autosomal dominant, autosomal recessive and X-linked recessive inheritance. Thirty families (Family $14,15,48,54,7$, $9,47,1,2,3,4,5,6,12,50,33,52,43,44,36,17,45,8,10,23,53,19,13,24$ and 31) with retinitis pigmentosa (or macular degeneration) had positive meaningful finding of gene mutations. With the help of genetic diagnosis, these patients were diagnosed as various types of retinitis pigmentosa. Some of these families acquired healthy offspring through genetic prenatal diagnosis or third generation test-tube infant technology (preimplantation genetic diagnosis). RP accounts for a large proportion of hereditary retinopathy in our study. It is difficult to distinguish cone-rod dystrophy from retinitis pigmentosa because of its similarity in clinical manifestations[13]. Through molecular genetic testing, we can make accurate diagnosis for patients with con-rod dystrophy (Family 34). It is difficult for some patients to differentiate choroideremia from RP, and detection of $\mathrm{CHM}$ gene mutation has differential diagnostic value. Choroideremia has a worse prognosis than RP[14]. The patient of Family 16 with choroideremia was diagnosed through targeted sequencing. Congenital stationary night blindness (CSNB) is similar with RP in clinical presentation, but the prognosis is better. We identified a case (Family 20) of CSNB, type 1C, through genetic targeting sequencing in this study.

Vitreoretinopathy is another major type of hereditary retinopathy. There are nine families (Family $18,32,46,55,35,37,49,25,56$ ) been detected gene mutations related to such diseases. Familial exudative vitreoretinopathy is a retinal vascular structural abnormality, with different inheritance patterns. The clinical symptoms of the disease vary greatly, even in the same family[15]. Mild patients have no symptoms, and only one disease related abnormality is a circular arc without vascular retina at the periphery of the terminal temporal area. The characteristics have been observed in these cases. Non-syndromic retinoschisis is an X-linked hereditary retinopathy, and its known pathogenic gene is RS1. We observed three different known pathogenic mutations of RS1 from three different families (Family 27, 38 and 51 ). The new mutation RS1 c. $240 \mathrm{G}>\mathrm{C}$ found in Family 26 may be benign, because both of the patient and his normal maternal grandfather carry the mutation. GoldmannFavre syndrome is an ocular syndrome with clinical symptoms including retinoschisis (Family 21).

Leber congenital amaurosis (LCA) is early onset and serious impairment of visual function[16]. Most of the patients may develop into blind children. Usually parents can observe visual abnormalities within one year of the child's birth. The children from four different families (Family 11 , 28,40 and 42) were diagnosed as LCA in our investigation. Targeted sequencing is of great significance in the diagnosis of hereditary ophthalmopathy. It will become part of our eye health management. We have also detected two cases of fundus developmental disease in our study, which are renal coloboma syndrome (Family 22) and foveal hypoplasia (Family 39). These two diseases are usually untreatable and have a general prognosis, but families with the disease could avoid high-risk offspring according to genetic rules. Two families (Family 41 and 29 ) with nystagmus have been detected two different mutations of GPR143 and FRMD7. The GPR143 or FRMD7 related nystagmus is X linked inheritance, with or without obvious retinal abnormalities. The genetic causes can guide these two families to have healthy offspring.

Previously, there were also studies that used Sanger method to sequence only one or several genes for molecular diagnosis of patients with different retinal disease[17-20]. One Bietti crystalline corneoretinal dystrophy research show us $84 \%$ detection rate by only CYP4V2 sequencing[17], and CYP4V2 is the only known pathogenic gene so far. It is likely to be a single example of over representation. The related detection rates of two familial exudative vitreoretinopathy studies are just 23\%[18] and 38.7\%[19] by sequenced three and six genes, respectively. Meaningful result of genetic testing usually requires a high level of accurate clinical diagnosis. However, retinal diseases have complex clinical manifestations and genetic heterogeneity. The clinical symptoms of some diseases are difficult to distinguish, and some diseases are related to more genes. As the number of genes needed to detect is increased, the efficiency of Sanger sequencing method becomes low. So, targeted sequencing becomes a better choice. Copy number variants analysis is also executed in this study without positive found, but exons duplication of OCRL has been found in Lowe syndrome in our previous work[21].

In summary, we researched the clinical and genetic characteristics of the families of hereditary retinopathy with positive findings on genetics level. We reported 37 novel related meaningful mutations and 31 known pathogenic variants for retinopathy, which lead to different relevant phenotypes of the eye diseases. These mutations are distributed in 31 different related genes. The diagnostic rate of LHON was $21.6 \%$ in our study, but no new mitochondrial pathogenic mutations were found. To our knowledge, this is a larger scale medical genetic study of retinal diseases in the Chinese population, and it reports many new pathogenic or significant related mutations and expands the phenotypic information of related diseases. Our study demonstrates the importance of studying a large collection of families with hereditary retinopathy due to the clinical manifestations and genetic heterogeneity of the diseases, which has guiding significance for this disease diagnosis and aristogenesis.

\section{Abbreviations}

Not applicable.

\section{Acknowledgements}

We would like to thank all the participants for their help and willingness to participate in this study. We thank the reviewers for the comments. We also thank the editor of this manuscript. This work was funded in part by National Key R\&D Program of China (2018YFC1002206-2). 


\section{References}

1. Xu, L., et al., Prevalence of retinitis pigmentosa in urban and rural adult Chinese: The Beijing Eye Study. Eur J Ophthalmol, 2006. 16(6): p. 8656.

2. Dimopoulos, I.S. and M. Xu, Re: Feuer et al.: Gene therapy for Leber hereditary optic neuropathy: initial results (Ophthalmology 2016;123:558570). Ophthalmology, 2017. 124(3): p. e22.

3. Petrs-Silva, H., et al., Suppression of rds expression by siRNA and gene replacement strategies for gene therapy using rAAV vector. Adv Exp Med Biol, 2012. 723: p. 215-23.

4. Ghazi, N.G., et al., Treatment of retinitis pigmentosa due to MERTK mutations by ocular subretinal injection of adeno-associated virus gene vector: results of a phase I trial. Hum Genet, 2016. 135(3): p. 327-43.

5. Haider, N.B., et al., Mutation of a nuclear receptor gene, NR2E3, causes enhanced S cone syndrome, a disorder of retinal cell fate. Nat Genet, 2000. 24(2): p. 127-31.

6. Marmor, M.F., A teenager with nightblindness and cystic maculopathy: enhanced S cone syndrome (Goldmann-Favre syndrome). Doc Ophthalmol, 2006. 113(3): p. 213-5.

7. Jespersgaard, C., et al., Molecular genetic analysis using targeted NGS analysis of 677 individuals with retinal dystrophy. Sci Rep, 2019. 9(1): p. 1219.

8. Martin-Merida, I., et al., Genomic Landscape of Sporadic Retinitis Pigmentosa: Findings from 877 Spanish Cases. Ophthalmology, 2019. 126(8): p. 1181-1188.

9. Stone, E.M., et al., Clinically Focused Molecular Investigation of 1000 Consecutive Families with Inherited Retinal Disease. Ophthalmology, 2017. 124(9): p. 1314-1331.

10. Carelli, V., et al., Parsing the differences in affected with LHON: genetic versus environmental triggers of disease conversion. Brain, 2016. 139(Pt 3): p. e17.

11. Black, G.C., et al., Leber's hereditary optic neuropathy: implications of the sex ratio for linkage studies in families with the 3460 ND1 mutation. Eye (Lond), 1995. 9 (Pt 4): p. 513-6.

12. Berson, E.L., Retinitis pigmentosa. The Friedenwald Lecture. Invest Ophthalmol Vis Sci, 1993. 34(5): p. $1659-76$.

13. Daiger, S.P., L.S. Sullivan, and S.J. Bowne, Genes and mutations causing retinitis pigmentosa. Clin Genet, 2013. 84(2): p. 132-41.

14. Chan, S.C., et al., Choroideremia research: Report and perspectives on the second international scientific symposium for choroideremia. Ophthalmic Genet, 2016. 37(3): p. 267-75.

15. Seo, S.H., et al., Molecular Characterization of FZD4, LRP5, and TSPAN12 in Familial Exudative Vitreoretinopathy. Invest Ophthalmol Vis Sci, 2015. 56(9): p. 5143-51.

16. Kumaran, N., et al., Leber congenital amaurosis/early-onset severe retinal dystrophy: clinical features, molecular genetics and therapeutic interventions. Br J Ophthalmol, 2017. 101(9): p. 1147-1154.

17. Yin, X., et al., Identification of CYP4V2 mutation in 36 Chinese families with Bietti crystalline corneoretinal dystrophy. Exp Eye Res, 2016. 146: p. $154-62$.

18. Tang, M., et al., Mutation Spectrum of the LRP5, NDP, and TSPAN12 Genes in Chinese Patients With Familial Exudative Vitreoretinopathy. Invest Ophthalmol Vis Sci, 2017. 58(13): p. 5949-5957.

19. Rao, F.Q., et al., Mutations in LRP5,FZD4, TSPAN12, NDP, ZNF408, or KIF11 Genes Account for 38.7\% of Chinese Patients With Familial Exudative Vitreoretinopathy. Invest Ophthalmol Vis Sci, 2017. 58(5): p. 2623-2629.

20. Alapati, A., et al., Molecular diagnostic testing by eyeGENE: analysis of patients with hereditary retinal dystrophy phenotypes involving central vision loss. Invest Ophthalmol Vis Sci, 2014. 55(9): p. 5510-21.

21. Bai, Z., et al., [Clinical and genetic analysis of an infant with Lowe syndrome caused by exonic duplication of OCRL gene]. Zhonghua Yi Xue Yi Chuan Xue Za Zhi, 2020. 37(1): p. 28-32.

22. Sung, C.H., et al., Rhodopsin mutations in autosomal dominant retinitis pigmentosa. Proc Natl Acad Sci U S A, 1991. 88(15): p. 6481-5.

23. Dryja, T.P., et al., Mutation spectrum of the rhodopsin gene among patients with autosomal dominant retinitis pigmentosa. Proc Natl Acad Sci U S A, 1991. 88(20): p. 9370-4.

24. Bunge, S., et al., Molecular analysis and genetic mapping of the rhodopsin gene in families with autosomal dominant retinitis pigmentosa. Genomics, 1993. 17(1): p. 230-3.

25. Shastry, B.S., J.F. Hejtmancik, and M.T. Trese, Identification of novel missense mutations in the Norrie disease gene associated with one Xlinked and four sporadic cases of familial exudative vitreoretinopathy. Hum Mutat, 1997. 9(5): p. 396-401.

26. Liu, D., et al., A novel nonsense mutation in the NDP gene in a Chinese family with Norrie disease. Mol Vis, 2010. 16: p. $2653-8$.

27. Smith, S.E., et al., Norrie disease: extraocular clinical manifestations in 56 patients. Am J Med Genet A, 2012. 158a(8): p. $1909-17$. 
28. Dai, H., et al., Identification of five novel mutations in the long isoform of the USH2A gene in Chinese families with Usher syndrome type II. Mol Vis, 2008. 14: p. 2067-75.

29. Katagiri, S., et al., Whole exome analysis identifies frequent CNGA1 mutations in Japanese population with autosomal recessive retinitis pigmentosa. PLoS One, 2014. 9(9): p. e108721.

30. Xu, W., et al., Seven novel mutations in the long isoform of the USH2A gene in Chinese families with nonsyndromic retinitis pigmentosa and Usher syndrome Type II. Mol Vis, 2011. 17: p. 1537-52.

31. Functional implications of the spectrum of mutations found in 234 cases with X-linked juvenile retinoschisis. The Retinoschisis Consortium. Hum Mol Genet, 1998. 7(7): p. 1185-92.

32. Huang, Y., et al., A novel deletion mutation in RS1 gene caused X-linked juvenile retinoschisis in a Chinese family. Eye (Lond), 2014. 28(11): p. 1364-9.

33. Xu, Y., et al., Mutations of 60 known causative genes in 157 families with retinitis pigmentosa based on exome sequencing. Hum Genet, 2014. 133(10): p. 1255-71.

34. Wada, Y., et al., Screening for mutations in CYP4V2 gene in Japanese patients with Bietti's crystalline corneoretinal dystrophy. Am J Ophthalmol, 2005. 139(5): p. 894-9.

35. Xiao, X., et al., Identification of CYP4V2 mutation in 21 families and overview of mutation spectrum in Bietti crystalline corneoretinal dystrophy. Biochem Biophys Res Commun, 2011. 409(2): p. 181-6.

36. Wada, Y., et al., Mutation of human retinal fascin gene (FSCN2) causes autosomal dominant retinitis pigmentosa. Invest Ophthalmol Vis Sci, 2001. 42(10): p. 2395-400.

37. Dong, B., et al., Two novel PRP31 premessenger ribonucleic acid processing factor 31 homolog mutations including a complex insertiondeletion identified in Chinese families with retinitis pigmentosa. Mol Vis, 2013. 19: p. 2426-35.

38. Yang, L., et al., Novel mutations of RPGR in Chinese retinitis pigmentosa patients and the genotype-phenotype correlation. PLoS One, 2014. 9(1): p. e85752.

39. Hosono, K., et al., Molecular Diagnosis of 34 Japanese Families with Leber Congenital Amaurosis Using Targeted Next Generation Sequencing. Sci Rep, 2018. 8(1): p. 8279.

40. Ernest, P.J., et al., Outcome of ABCA4 microarray screening in routine clinical practice. Mol Vis, 2009. 15: p. $2841-7$.

41. Lewis, R.A., et al., Genotype/Phenotype analysis of a photoreceptor-specific ATP-binding cassette transporter gene, ABCR, in Stargardt disease. Am J Hum Genet, 1999. 64(2): p. 422-34.

42. Wang, H., et al., Comprehensive Molecular Diagnosis of a Large Chinese Leber Congenital Amaurosis Cohort. Invest Ophthalmol Vis Sci, 2015. 56(6): p. 3642-55.

43. Gerber, S., et al., Complete exon-intron structure of the RPGR-interacting protein (RPGRIP1) gene allows the identification of mutations underlying Leber congenital amaurosis. Eur J Hum Genet, 2001. 9(8): p. 561-71.

44. van Genderen, M.M., et al., Mutations in TRPM1 are a common cause of complete congenital stationary night blindness. Am J Hum Genet, 2009. 85(5): p. 730-6.

45. Negrisolo, S., et al., PAX2 gene mutations in pediatric and young adult transplant recipients: kidney and urinary tract malformations without ocular anomalies. Clin Genet, 2011. 80(6): p. 581-5.

46. Lenis, T.L., et al., Novel compound heterozygous mutations resulting in cone dystrophy with supernormal rod response. JAMA Ophthalmol, 2013. 131(11): p. 1482-5.

47. Poulter, J.A., et al., Recessive mutations in SLC38A8 cause foveal hypoplasia and optic nerve misrouting without albinism. Am J Hum Genet, 2013. 93(6): p. 1143-50.

48. Li, N., et al., Five novel mutations of the FRMD7 gene in Chinese families with X-linked infantile nystagmus. Mol Vis, 2008. 14: p. 733-8.

49. Chen, X., et al., Targeted sequencing of 179 genes associated with hereditary retinal dystrophies and 10 candidate genes identifies novel and known mutations in patients with various retinal diseases. Invest Ophthalmol Vis Sci, 2013. 54(3): p. $2186-97$.

50. Aparisi, M.J., et al., Targeted next generation sequencing for molecular diagnosis of Usher syndrome. Orphanet J Rare Dis, 2014 . 9: p. 168.

51. McGee, T.L., et al., Novel mutations in the long isoform of the USH2A gene in patients with Usher syndrome type II or non-syndromic retinitis pigmentosa. J Med Genet, 2010. 47(7): p. 499-506.

52. Redin, C., et al., Targeted high-throughput sequencing for diagnosis of genetically heterogeneous diseases: efficient mutation detection in Bardet-Biedl and Alstrom syndromes. J Med Genet, 2012. 49(8): p. 502-12.

53. Qin, M., et al., Complexity of the genotype-phenotype correlation in familial exudative vitreoretinopathy with mutations in the LRP5 and/or FZD4 genes. Hum Mutat, 2005. 26(2): p. 104-12.

54. Jiang, F., et al., Screening of ABCA4 Gene in a Chinese Cohort With Stargardt Disease or Cone-Rod Dystrophy With a Report on 85 Novel Mutations. Invest Ophthalmol Vis Sci, 2016. 57(1): p. 145-52.

Page $8 / 13$ 


\section{Tables}

Table 1. General situation of families with pathogenic or likely pathogenic mutation 


\begin{tabular}{|c|c|c|c|c|c|c|c|c|c|c|c|c|}
\hline$\underline{\underline{\mathrm{Fa}}}$ & $\underline{\underline{N p}}$ & $\underline{\text { Gene }}$ & Transcript & $\underline{\underline{E x}}$ & NA Changes & AA changes & $\underline{\text { Hzyo }}$ & $\underline{\underline{\mathrm{Pf}}}$ & Reported & $\underline{\mathrm{Gm}}$ & Disease & SPM \\
\hline 14 & 2 & RHO & NM_000539 & 1 & c. $251 \mathrm{~T}>\mathrm{C}$ & p.L84P & Het & - & Novel & $\mathrm{AD}$ & $\mathrm{RP}, 4$ & +++ \\
\hline 15 & 4 & RHO & NM_000539 & 2 & c. $403 \mathrm{C}>\mathrm{T}$ & p.R135W & Het & $\begin{array}{c}0 / \\
1.082^{\mathrm{e}-5} \\
\end{array}$ & Yes[22] & $\mathrm{AD}$ & $\mathrm{RP}, 4$ & +++ \\
\hline 48 & 1 & RHO & NM_000539 & 3 & c. $541 \mathrm{G}>\mathrm{A}$ & p.E181K & Het & - & Yes[23] & $\mathrm{AD}$ & $\mathrm{RP}, 4$ & +++ \\
\hline 54 & 2 & RHO & NM_000539 & 2 & c. $403 \mathrm{C}>\mathrm{T}$ & p.R135W & Het & $\begin{array}{c}0 / \\
1.082^{\mathrm{e}-5} \\
\end{array}$ & Yes[24] & $\mathrm{AD}$ & $\mathrm{RP}, 4$ & +++ \\
\hline 18 & 3 & $N D P$ & NM_000266 & 2 & c. $124 \mathrm{C}>\mathrm{A}$ & p.H42N & Hemi & - & Novel & $\mathrm{XL}$ & FEVR2[25] & +++ \\
\hline 32 & 1 & $N D P$ & NM_000266 & 3 & c. $343 \mathrm{C}>\mathrm{T}$ & p.R115X & Hemi & - & Yes[26] & XLR & Norrie & +++ \\
\hline 46 & 1 & $N D P$ & NM_000266 & 3 & c.401_402delGA & p.*134Wfs*13 & Hemi & - & Novel & $\mathrm{XL}$ & FEVR2 & 111 \\
\hline 55 & 3 & $N D P$ & NM_000266 & 3 & c. $268 \mathrm{C}>\mathrm{T}$ & p.R90C & Hemi & - & Yes[27] & XLR & Norrie & +++ \\
\hline 7 & 1 & USH $2 A$ & NM_206933 & 2 & c.99_100insT & p.R34Sfs*41 & Hom & $\begin{array}{c}6.242^{\mathrm{e}-} \\
5 / \\
3.231^{\mathrm{e}-5} \\
\end{array}$ & Yes[28] & $\mathrm{AR}$ & Usher 2A & $1 / 1$ \\
\hline \multirow[t]{2}{*}{9} & \multirow[t]{2}{*}{1} & USH $2 A$ & NM_206933 & 55 & c. $10859 \mathrm{~T}>\mathrm{C}$ & p.I3620T & Het & $\begin{array}{l}1.16^{\mathrm{e}-4} / \\
1.219^{\mathrm{e}-5} \\
\end{array}$ & Yes[29] & $\mathrm{AR}$ & $\begin{array}{l}\text { Usher } 2 \mathrm{~A} / \mathrm{RP}, \\
39 \\
\end{array}$ & +++ \\
\hline & & USH2A & NM_206933 & 13 & c. $2802 \mathrm{~T}>\mathrm{G}$ & p.C934W & Het & $\begin{array}{c}2.441^{\mathrm{e}-3} / \\
1.915^{\mathrm{e}-4}\end{array}$ & Yes[30] & $\mathrm{AR}$ & $\begin{array}{c}\text { Usher } 2 \mathrm{~A} / \mathrm{RP}, \\
39 \\
\end{array}$ & +++ \\
\hline \multirow[t]{2}{*}{47} & \multirow[t]{2}{*}{1} & USH $2 A$ & NM_206933 & 63 & c.13596dupC & p.S4533Lfs*28 & Het & - & Novel & $\mathrm{AR}$ & Usher 2A & 111 \\
\hline & & USH2A & NM_206933 & 56 & c. $10962 \mathrm{C}>\mathrm{A}$ & p.Y3654X & Het & - & Novel & $\mathrm{AR}$ & Usher $2 \mathrm{~A}$ & +++ \\
\hline 27 & 1 & $R S 1$ & NM_000330 & 6 & c. $598 \mathrm{C}>\mathrm{T}$ & p.R200C & Hemi & - & Yes[31] & XLR & Retinoschisis & +++ \\
\hline 38 & 1 & $R S 1$ & NM_000330 & 4 & c. $214 \mathrm{G}>\mathrm{A}$ & p.E72K & Hemi & $\begin{array}{c}0 / \\
1.678^{\mathrm{e}-5} \\
\end{array}$ & Yes[31] & XLR & Retinoschisis & +++ \\
\hline 51 & 2 & $R S 1$ & NM_000330 & 4 & c.206_207delTG & p.Leu69Argfs*16 & Hemi & - & Yes[32] & XLR & Retinoschisis & 111 \\
\hline \multirow[t]{2}{*}{1} & \multirow[t]{2}{*}{1} & MERTK & NM_006343 & 8 & c. $1186 \mathrm{G}>\mathrm{T}$ & p.E396X & Het & - & Yes[33] & $\mathrm{AR}$ & $\mathrm{RP}, 38$ & $11+$ \\
\hline & & MERTK & NM_006343 & 3 & c. $518 \mathrm{~A}>\mathrm{G}$ & p.Y173C & Het & $\begin{array}{c}0 / \\
1.219^{\mathrm{e}-5} \\
\end{array}$ & Novel & $\mathrm{AR}$ & $\mathrm{RP}, 38$ & +++ \\
\hline 2 & 2 & MERTK & NM_006343 & 4 & c.754delC & p.P252Qfs*3 & Hom & - & Novel & $\mathrm{AR}$ & $\mathrm{RP}, 38$ & 111 \\
\hline 3 & 1 & $C Y P 4 V 2$ & NM_207352 & 7 & $\begin{array}{c}\text { c.(802- } \\
\text { 8)_810delTCATACAGGTCATCGCTinsGC }\end{array}$ & $\begin{array}{l}\text { ?p.268_270del/ } \\
\text { Splicing }\end{array}$ & Hom & $\begin{array}{l}7.963^{\mathrm{e}-4} / \\
6.856^{\mathrm{e}-5} \\
\end{array}$ & Yes[34] & $\mathrm{AR}$ & Bietti CCD & $1 / 1$ \\
\hline \multirow[t]{2}{*}{4} & \multirow[t]{2}{*}{2} & $C Y P 4 V 2$ & NM_207352 & 7 & $\begin{array}{c}\text { c.(802- } \\
\text { 8)_810delTCATACAGGTCATCGCTinsGC }\end{array}$ & $\begin{array}{l}\text { ?p.268_270del/ } \\
\text { Splicing }\end{array}$ & Het & $\begin{array}{l}7.963^{\mathrm{e}-4} / \\
6.856^{\mathrm{e}-5} \\
\end{array}$ & Yes[34] & $\mathrm{AR}$ & Bietti CCD & $1 / 1$ \\
\hline & & $C Y P 4 V 2$ & NM_207352 & 7 & c.958C $>\mathrm{T}$ & p.R320X & Het & $\begin{array}{c}0 / \\
4.061^{\mathrm{e}-6} \\
\end{array}$ & Yes[35] & $\mathrm{AR}$ & Bietti CCD & $11+$ \\
\hline 5 & 4 & FSCN2 & NM_001077182 & 1 & c.72delG & p.T25Qfs*120 & Het & $\begin{array}{c}0.01238 / \\
8.801^{\mathrm{e}-4} \\
\end{array}$ & Yes & $\mathrm{AD}$ & $\mathrm{RP}, 30$ & $1 / 1$ \\
\hline 6 & 2 & FSCN2 & NM_001077182 & 1 & c.72delG & p.T25Qfs*120 & Het & $\begin{array}{c}0.01238 / \\
8.801^{\mathrm{e}-4}\end{array}$ & Yes[36] & $\mathrm{AD}$ & $\mathrm{RP}, 30$ & $1 / 1$ \\
\hline 12 & 4 & PRPF31 & NM_015629 & 11 & c.(1074-8)_1079delGTCCCCAGGTACCG & $\begin{array}{c}\text { ? } \\
\text { p.358_360delRYRinsS/ } \\
\text { Splicing } \\
\end{array}$ & Het & - & Novel & $\mathrm{AD}$ & $\mathrm{RP}, 11$ & $1 / 1$ \\
\hline 50 & 2 & PRPF31 & NM_015629 & 12 & c.1215delG & p.Val406fs*7 & Het & - & Yes[37] & $\mathrm{AD}$ & $\mathrm{RP}, 11$ & $1 / 1$ \\
\hline 33 & 1 & $R P G R$ & NM_001034853 & 15 & c.2236_2237delGA & p.E746Rfs*23 & Hemi & - & Yes & XLR & $\mathrm{MD}$ & 111 \\
\hline 52 & 2 & $R P G R$ & NM_001034853 & 15 & c.2236_2237delGA & p.E746Rfs*23 & Hemi & - & Yes[38] & XLR & $\mathrm{MD}$ & 111 \\
\hline 43 & 5 & $R P 2$ & NM_006915 & 3 & c. $769-2 \mathrm{~A}>\mathrm{G}$ & splicing & Hemi & - & Yes[39] & $\mathrm{XL}$ & $\mathrm{RP}, 2$ & $11+$ \\
\hline 44 & 4 & $R P 2$ & NM_006915 & 2 & c.572_582dup11 & p.Pro190Profs*52 & Hemi & - & Novel & $\mathrm{XL}$ & $\mathrm{RP}, 2$ & 111 \\
\hline \multirow[t]{2}{*}{36} & 1 & $A B C A 4$ & NM_000350 & 29 & c. $4352+1 \mathrm{G}>\mathrm{A}$ & splicing & Het & $\begin{array}{c}0 / \\
8.123^{\mathrm{e}-6} \\
\end{array}$ & Yes[40] & $\mathrm{AR}$ & Stargardt 1 & $11+$ \\
\hline & & $A B C A 4$ & NM_000350 & 13 & c. $1804 \mathrm{C}>\mathrm{T}$ & p.R602W & Het & $\begin{array}{l}2.904^{\mathrm{e}-4} / \\
4.477^{\mathrm{e}-5}\end{array}$ & Yes[41] & $\mathrm{AR}$ & Stargardt 1 & +++ \\
\hline \multirow[t]{2}{*}{11} & 1 & TULP1 & NM_003322 & 13 & c. $1318 \mathrm{C}>\mathrm{T}$ & p.R440X & Het & $\begin{array}{c}0 / \\
1.145^{\mathrm{e}-5} \\
\end{array}$ & Yes[42] & $\mathrm{AR}$ & LCA 15 & $1 /+$ \\
\hline & & TULP1 & NM_003322 & 12 & c. $1142 \mathrm{~T}>\mathrm{G}$ & p.V381G & Het & - & Novel & $\mathrm{AR}$ & LCA 15 & +++ \\
\hline 16 & 1 & $\mathrm{CHM}$ & NM_000390 & 5 & c.544delT & p.C182Vfs*14 & Hemi & - & Novel & XLD & choroideremia & 111 \\
\hline 28 & 1 & RPGRIP1 & NM_020366 & 16 & c. $2662 \mathrm{C}>\mathrm{T}$ & p.R888X & Hom & $0 / 1.68^{\mathrm{e}-}$ & Yes[43] & $\mathrm{AR}$ & LCA6 & +++ \\
\hline 17 & 2 & PRPF8 & NM_006445 & 36 & c. $5792 \mathrm{C}>\mathrm{T}$ & p.T1931M & Het & - & Novel & $\mathrm{AD}$ & $\mathrm{RP}, 13$ & +++ \\
\hline \multirow[t]{2}{*}{20} & \multirow[t]{2}{*}{1} & TRPM1 & $\begin{array}{c}\text { NM_0012 } \\
52020 \\
\end{array}$ & 21 & c. $2789 \mathrm{~T}>\mathrm{A}$ & p.I930N & Het & - & Novel & $\mathrm{AR}$ & CSNB1C & +++ \\
\hline & & TRPM1 & $\begin{array}{c}\text { NM_0012 } \\
52020 \\
\end{array}$ & 22 & c. $3178+1 \mathrm{G}>\mathrm{A}$ & splicing & Het & $\begin{array}{l}6.889^{\mathrm{e}-4} / \\
5.772^{\mathrm{e}-5}\end{array}$ & Yes[44] & $\mathrm{AR}$ & CSNB1C & $11+$ \\
\hline 21 & 1 & NR2E3 & NM_014249 & 6 & c. $925 \mathrm{C}>\mathrm{T}$ & p.R309W & Hom & $\begin{array}{c}0 / 8.34^{\mathrm{e}-} \\
6\end{array}$ & Novel & $\mathrm{AR}$ & GF & $1+1$ \\
\hline 22 & 1 & $P A X 2$ & NM_003990 & 2 & c.70dupG & p.V26Gfs*28 & Het & $\begin{array}{c}0 / \\
1.237^{\mathrm{e}-5} \\
\end{array}$ & Yes[45] & $\mathrm{AD}$ & RCS & $1 / 1$ \\
\hline \multirow[t]{2}{*}{34} & \multirow[t]{2}{*}{2} & KCNV2 & NM_133497 & 1 & c.506_513delTGCTGCT & p.V169Gfs*40 & Het & - & Novel & $\mathrm{AR}$ & RCD3B & 111 \\
\hline & & KCNV2 & NM_133497 & 1 & c.137G $>A$ & p.W46X & Het & - & Yes[46] & $\mathrm{AR}$ & RCD3B & +++ \\
\hline 35 & 2 & FZD4 & NM_206933 & 2 & c. $612 \mathrm{~T}>\mathrm{A}$ & p.C204X & Het & - & Novel & $\mathrm{AD}$ & FEVR1 & +++ \\
\hline 37 & 2 & LRP5 & NM_002335 & 2 & c.485_488delACGG & p.H162Rfs*38 & Het & - & Novel & $\mathrm{AD}$ & FEVR4 & 111 \\
\hline \multirow[t]{2}{*}{39} & \multirow[t]{2}{*}{1} & SLC38A8 & NM_001080442 & 7 & c.927_928delCT & p.Y310Pfs*57 & Het & - & Novel & $\mathrm{AR}$ & FH2 & 111 \\
\hline & & $S L C 38 A 8$ & NM_001080442 & 6 & c. $697 \mathrm{G}>\mathrm{A}$ & p.E233K & Het & $\begin{array}{c}2.778^{\mathrm{e}-4} / \\
6.886^{\mathrm{e}-5} \\
\end{array}$ & Yes[47] & $\mathrm{AR}$ & FH2 & +++ \\
\hline 40 & 1 & AIPL1 & NM_001285399 & 3 & c. $385 \mathrm{C}>\mathrm{T}$ & p.Q129X & Hom & - & Novel & $\mathrm{AR}$ & LCA4 & +++ \\
\hline 41 & 1 & FRMD7 & NM_194277 & 10 & c. $.910 \mathrm{C}>\mathrm{T}$ & p.R304X & Hemi & $\begin{array}{c}0 / \\
5.608^{\mathrm{e}-6} \\
\end{array}$ & Yes[48] & XLR & Nystagmus 1 & +++ \\
\hline 42 & 1 & GUCY2D & NM_000180 & 18 & c.3177_3178delAC & p.R1060Rfs*11 & Hom & $\begin{array}{c}0 / \\
4.935^{\mathrm{e}-6} \\
\end{array}$ & Novel & $\mathrm{AR}$ & LCA4 & 111 \\
\hline 45 & 1 & $C N G A 1$ & NM_001142564 & 5 & c. $472 \mathrm{delC}$ & p.L158Ffs*4 & Het & $\begin{array}{l}0.0012 / \\
6.455^{\mathrm{e}-5}\end{array}$ & Novel[49] & $\mathrm{AR}$ & $\mathrm{RP}, 49$ & 111 \\
\hline & & CNGA1 & NM_001142564 & 5 & c. $453 \mathrm{C}>\mathrm{A}$ & p.Y151X & Het & $5.798^{\mathrm{e}-5} /$ & Novel & $\mathrm{AR}$ & $\mathrm{RP}, 49$ & +++ \\
\hline
\end{tabular}




\begin{tabular}{|c|c|c|c|c|c|c|c|c|c|c|c|c|}
\hline & & & & & & & & $4.068^{\mathrm{e}-6}$ & & & & \\
\hline 49 & 3 & TSPAN12 & NM_012338 & 8 & $\begin{array}{l}\text { c.731delT } \\
\end{array}$ & p.L244Rfs*17 & Het & $\begin{array}{c}0 / \\
4.064^{\mathrm{e}-6}\end{array}$ & Novel & $\mathrm{AD}$ & EV5 & 111 \\
\hline
\end{tabular}

Table 2. General situation of families with likely pathogenic mutations or related mutations of Undetermined Significance

\begin{tabular}{|c|c|c|c|c|c|c|c|c|c|c|c|c|}
\hline$\underline{\underline{\mathrm{Fa}}}$ & $\underline{\underline{N p}}$ & Gene & Transcript & $\underline{\underline{E x}}$ & NA Changes & AA changes & $\underline{\underline{\text { Hzyo }}}$ & $\underline{\underline{P f}}$ & Reported & $\underline{\mathrm{Gm}}$ & $\underline{\text { OMIM Disease }}$ & SPM \\
\hline \multirow[t]{2}{*}{8} & \multirow[t]{2}{*}{1} & USH $2 A$ & NM_206933 & 41 & c. $8002 \mathrm{G}>\mathrm{T}$ & p.E2668X & Het & - & Novel & $\mathrm{AR}$ & Usher 2A/RP, 39 & $11+$ \\
\hline & & USH2A & NM_206933 & 13 & c. $2802 \mathrm{~T}>\mathrm{G}$ & p.C934W & Het & $2.441^{\mathrm{e}-3} / 1.915^{\mathrm{e}-4}$ & Yes [30] & $\mathrm{AR}$ & Usher $2 \mathrm{~A} / \mathrm{RP}, 39$ & +++ \\
\hline \multirow[t]{2}{*}{10} & \multirow[t]{2}{*}{1} & USH2A & NM_206933 & 63 & C. $12608 \mathrm{~A}>\mathrm{G}$ & p.Q4203R & Het & $9.457^{\mathrm{e}-3} / 3.677^{\mathrm{e}-3}$ & Novel & $\mathrm{AR}$ & Usher 2A/RP, 39 & - - \\
\hline & & USH2A & NM_206933 & 22 & c. $4758+3 A>G$ & Splicing & $\mathrm{Het}$ & $1.855^{\mathrm{e}-2} / 1.457^{\mathrm{e}-3}$ & Yes [50] & $\mathrm{AR}$ & Usher 2A/RP, 39 & 111 \\
\hline \multirow[t]{2}{*}{23} & \multirow[t]{2}{*}{1} & USH2A & NM_206933 & 66 & c. $14411 \mathrm{G}>\mathrm{A}$ & p.G4804E & Het & - & Novel & $\mathrm{AR}$ & Usher 2A/RP, 39 & $11+$ \\
\hline & & USH2A & NM_206933 & 19 & c. $4217 \mathrm{C}>\mathrm{A}$ & p.S1406X & Het & - & Novel & $\mathrm{AR}$ & Usher 2A/RP, 39 & +++ \\
\hline \multirow[t]{2}{*}{53} & \multirow[t]{2}{*}{1} & USH2A & NM_206933 & 65 & c. $14287 \mathrm{G}>\mathrm{A}$ & p.G4763R & Het & - & Yes[51] & $\mathrm{AR}$ & Usher $2 \mathrm{~A} / \mathrm{RP}, 39$ & +++ \\
\hline & & USH $2 A$ & NM_206933 & 4 & c. $784+2 \mathrm{~T}>\mathrm{G}$ & Splicing & Het & - & Novel & $\mathrm{AR}$ & Usher 2A/RP, 39 & 111 \\
\hline \multirow[t]{2}{*}{19} & \multirow[t]{2}{*}{1} & USH1C & NM_153676 & 5 & c. $407 \mathrm{G}>\mathrm{A}$ & p.R136Q & Het & $1.16^{\mathrm{e}-4} / 1.223^{\mathrm{e}-4}$ & Novel & $\mathrm{AR}$ & Usher 1C & $11+$ \\
\hline & & USH1C & NM_153676 & 15 & c. $1250 \mathrm{C}>\mathrm{T}$ & p.T417I & Het & - & Novel & $\mathrm{AR}$ & Usher 1C & $11+$ \\
\hline \multirow[t]{2}{*}{13} & \multirow[t]{2}{*}{1} & $B B S 2$ & NM_031885 & 6 & c. $626 \mathrm{~T}>\mathrm{C}$ & p.L209P & Het & - & Yes [52] & $\mathrm{AR}$ & $\mathrm{RP}, 74$ & +++ \\
\hline & & $B B S 2$ & NM_031885 & 1 & c. $79 A>C$ & p.T27P & Het & - & Novel & $\mathrm{AR}$ & $\mathrm{RP}, 74$ & - - \\
\hline \multirow[t]{2}{*}{25} & \multirow[t]{2}{*}{1} & $L R P 5$ & NM_002335 & 15 & c. $3361 \mathrm{~A}>\mathrm{G}$ & p.N1121D & Het & $7.528^{\mathrm{e}-3} / 5.616^{\mathrm{e}-4}$ & Yes [53] & $\mathrm{AR}$ & FEVR4 & +++ \\
\hline & & $L R P 5$ & NM_002335 & 18 & c. $3901 \mathrm{G}>\mathrm{A}$ & p.A1301T & Het & $2.403^{\mathrm{e}-3} / 2.149^{\mathrm{e}-4}$ & Novel & $\mathrm{AR}$ & FEVR4 & $\ldots$ \\
\hline \multirow[t]{2}{*}{56} & \multirow[t]{2}{*}{1} & $L R P 5$ & NM_002335 & 15 & c. $3377 \mathrm{~T}>\mathrm{C}$ & p.L1126P & Het & - & Novel & $\mathrm{AR}$ & FEVR4 & +++ \\
\hline & & $L R P 5$ & NM_002335 & 22 & c. $4519 \mathrm{G}>\mathrm{T}$ & p.D1507T & Het & - & Novel & $\mathrm{AR}$ & FEVR4 & +++ \\
\hline 24 & 1 & $A B C A 4$ & NM_000350 & 5 & c. $553 \mathrm{C}>\mathrm{T}$ & p.Q185X & Het & - & Yes [54] & $\mathrm{AD}$ & AMD2 & +++ \\
\hline 26 & 1 & $R S 1$ & NM_000330 & 4 & c. $240 \mathrm{G}>\mathrm{C}$ & p.Q80H & Hemi & - & Novel & XLR & Retinoschisis & $11+$ \\
\hline 29 & 1 & GPR143 & NM_000273 & 2 & c. $263 \mathrm{G}>\mathrm{A}$ & p.R88Q & Hemi & - & Novel & $\mathrm{XL}$ & Nystagmus 6 & +++ \\
\hline 31 & 1 & $F B N 2$ & NM_001999 & 30 & c.3923dupG & p.C1308Wfs*5 & Het & - & Novel & $\mathrm{AD}$ & EMD & 111 \\
\hline
\end{tabular}

Notes for Table 1 and 2: Fa short for Family No.; Np short for number of patients; Ex short for exon; NA short for nucleic acid; AA short for amino acid; Hzyo short for heterozygosity; Pf short for population frequency recorded in gnomAD database; Gm short for genetic model; Disease stands for OMIM disease; SPM short for SIFT, PolyPhen_2 and Mutation t@sting predicting, '+' stands for damaging, '-' stands for benign and '/' stands for no data.

RP, 38 stands for retinitis pigmentosa, type 38; Bietti CCD stands for Bietti crystalline corneoretinal dystrophy; RP, 30 stands for retinitis pigmentosa, type 30; Usher 2A stands for Usher syndrome, type 2A; Usher 1C stands for Usher syndrome, type 1C; LCA 15 stangds for Leber congenital amaurosis, type 15; RP, 11 stands for retinitis pigmentosa, type 11; RP,4 stands for retinitis pigmentosa, type 4; RP, 13 stands for retinitis pigmentosa, type 13; FEVR2 stands for familial exudative vitreoretinopathy, type 2; CSNB1C stands for congenital stationary night blindness, type 1C; GF stands for Goldmann-Favre syndrome; RCS stands for renal coloboma syndrome; FEVR1 stands for familial exudative vitreoretinopathy, type 1; Stargardt 1 stands for Stargardt's disease, type1; FEVR4 stands for familial exudative vitreoretinopathy, type 4; FH2 stands for foveal hypoplasia, type 2; LCA4 stangds for Leber congenital amaurosis, type 4; MD stands for macular degeneration, X-linked atrophic; RCD3B stands for retinal cone dystrophy, type 3B; Nystagmus 1 stands for nystagmus, type 1, congenital, X-linked; RP, 2 stands for retinitis pigmentosa, type 2; RP,74 stands for retinitis pigmentosa, type 74; AMD2 stands for age-related macular degeneration, type 2; FEVR4 stands for familial exudative vitreoretinopathy, type4; LCA6 stangds for Leber congenital amaurosis, type 6; Nystagmus 6 stangds for nystagmus, type 6, congenital, X-linked; EMD stands for macular degeneration, early-onset; RP,39 stands for retinitis pigmentosa, type 39; EV5 stands for exudative vitreoretinopathy, type 5

\section{Declarations}

- Ethics approval and consent to participate-- Clinical investigations were conducted according to the Declaration of Helsinki, and the study was approved by the institutional review board of the Medical Ethics Committee of the First affiliated Hospital of Zhengzhou University. For all the minor patients, their parents agree their participation in this study. The adult patients also consented to participate in this study.

- Consent for publication- Written informed consent for publication of clinical details and/or clinical images was obtained from all of the participants. Informed consent of individuals younger than the age of 18 was written by their parents.

- Competing interests-- The authors report no conflicts of interest.

- Availability of data and materials- The datasets used and analysed during the current study are available from the corresponding author on reasonable request.

\section{Figures}




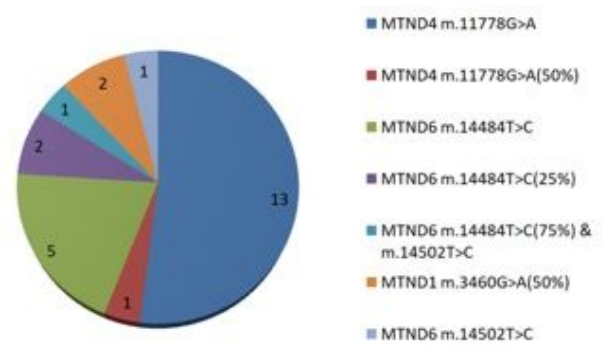

Figure 1

Variation distribution of 25 patients with Leber's hereditary optic neuropathy $₫ 21.6 \% \rrbracket$

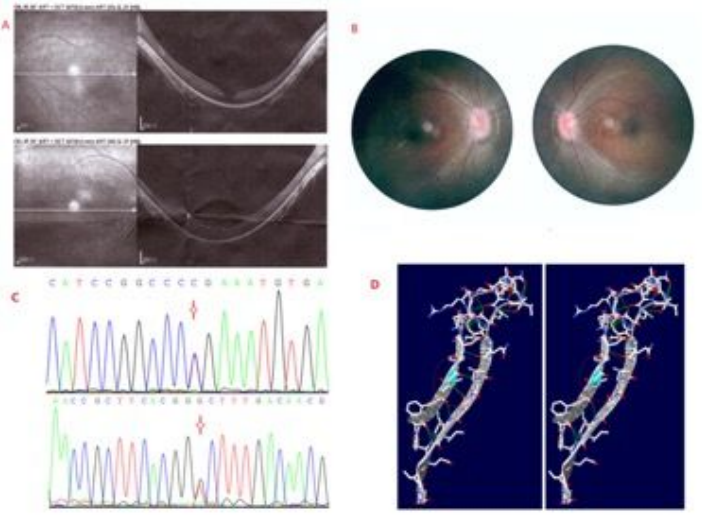

Figure 2

TULP1 mutations and clinical manifestation of the LCA 15 patient in Family 11. Part A, optical coherence tomography (OCT) shows that the temporal retinal neuroepithelium of macula of both eyes became thinning, the central fovea of macula formed backward concave; Part $\mathrm{B}$, ophthalmoscopic examination shows that the boundary of optic disc is blurred and the retina is dark; Part C, the compound heterozygous mutation of TULP1 c.1318C>T p.R440X (above) and c.1142T>G p.V381G(below); Part D, Secondary structure change of the novel mutation, TULP1 c. $1142 T>$ G.
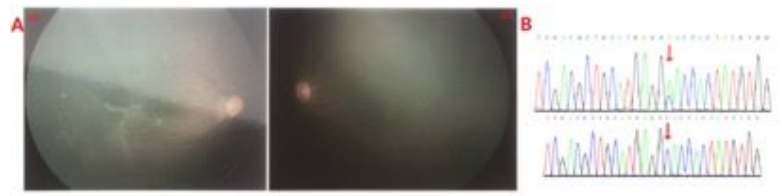

\section{Figure 3}

NDP c.124C>A hemizygous mutation and fundus avascular area of the FEVR2 patient in Family 18. Part A, fundus examination of the one year old patient showed that there was avascular area in both eyes. The temporal side of the blood vessel arch in the right eye fundus showed the epiretinal membrane and the macular traction. Part B, NDP c.124C>A mutation of the mother and the child, respectively.
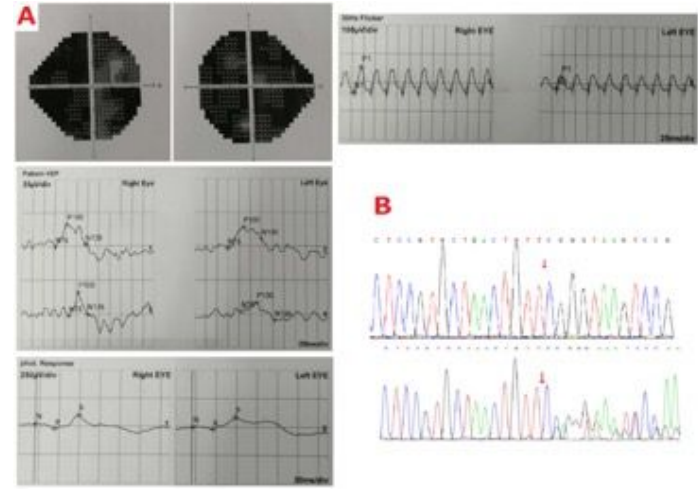

B

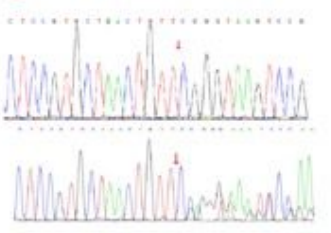

Figure 4 
Part A, the older sister with binocular patchy visual field in Family 2, Her P-VEP examination showed bilateral P100 wave latency delay with normal amplitude. F-ERG examination showed that binocular light adaptation, $30 \mathrm{~Hz}$ response amplitude moderately or severely decreased, other response amplitude moderately decreased; binocular dark response could not be induced, OPS wavelets could not be separated, other waves could be induced and the amplitude moderately decreased. Part B is the Sanger sequencing results of the mutated site.

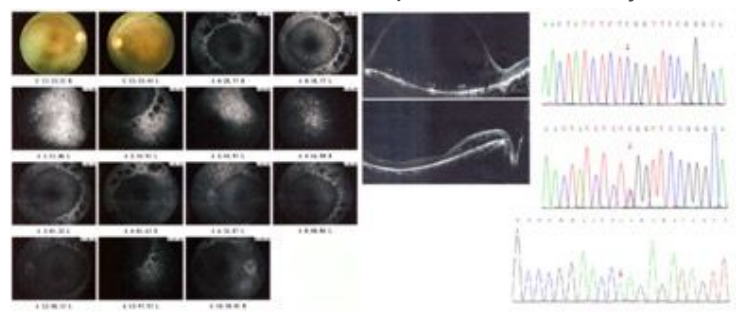

Figure 5

Fluorescein fundus angiography examination of patient in family 21 showed that the filling time of bilateral arteriovenous fluorescence was prolonged. In the early stage, inhomogeneous strong fluorescence and occluded fluorescence could be seen in the posterior pole of both eyes. Strong fluorescence and inhomogeneous fluorescence could also be seen in the periphery. In the late stage, inhomogeneous strong fluorescence could be seen in the periphery of both eyes, and no obvious fluorescence leakage could be observed. OcT examination showed that the nerve cortex in macular area of left eye splitted, the fovea in macular area was not seen, and the pigmented epithelium was rough; the fovea in macular area of right eye was not obvious, the pigmented epithelium in macular area was rough, and the nasal retinal layer splitted.

\section{Supplementary Files}

This is a list of supplementary files associated with this preprint. Click to download.

- SupplementaryMaterialGenomics.docx 